\title{
A case of recurrent ventriculitis associated with Colistin-Resistant Klebsiella pneumoniae in patient with ventriculoperitoneal shunt treated with intrathecal amikacin
}

\author{
Abdurrahman Kaya $^{1 *}$, İlker İnanç Balkan ${ }^{1}$, Sibel Yıldız Kaya ${ }^{1}$, Berna Altun ${ }^{1}$, Mücahit Yemişen ${ }^{1}$, Bilgül Mete ${ }^{1}$, Neşe Saltoğlu ${ }^{1}$, Yusuf Tunalı ${ }^{2}$, \\ Gökhan Aygün ${ }^{1}$ and Recep Öztürk ${ }^{1}$ \\ ${ }^{1}$ Department of Infectious Diseases, İstanbul Training and Research Hospital, Istanbul, Turkey \\ ${ }^{2}$ Department of Anesthesiology and Intensive Care, Medical school of cerrahpaşa, Istanbul, Turkey
}

\begin{abstract}
Carbapenem-resistant Klebsiella pneumoniae (CR-KP) strains have been increasingly seen as agents in the nosocomial infections in recent years. Many drugs, especially colistin have been mostly used in the treatment of these pathogens. With the increasing use of colistin, we have been encountered colistin-resistant strains in the hospital outbreak and infections. The treatment of carbapenemase-producing organisms is unclear and has not been reached a consensus yet. Two or more antibiotics have been administered due to high mortality rate and a major concern in emergence of new resistance. Herein, we presented a patient who was administered intravenous (IV) + intrathecal colistin therapy due to recurrent ventriculitis associated with ventriculoperitoneal (VP) shunt. But the condition of the patient worsened under this treatment and then colistin-resistant Klebsiella pneumoniae (CoR-KP) was isolated from cerebrospinal fluid (CSF) culture. Finally, she could be successfully treated with intravenous and intrathecal amikacin use. Resistant bacteria seem to increase and pose a threat to our life in the future. Thus, infection control measures should be taken immediately. In these infections, along with the use of systemic antibiotics, intrathecal use should be administered for meningitidis.
\end{abstract}

\section{Introduction}

Carbapenem-resistant Klebsiella pneumoniae (CR-KP) strains have been increasingly seen as agents in the nosocomial infections in recent years. Many drugs, especially colistin are mostly used in the treatment of these pathogens [1]. As a natural result, we encountered colistinresistant strains in the hospital outbreak and infections. This challenge provide very limited treatment options for us and it shows that not only carbapenem but also colistin need to be used appropriately because of possibility of resistance [2]. Antibiotic resistance is seen due to unnecessarily and inappropriately using and not implementing hospital infection control procedures. Herein, we presented a patient who was administered intravenous (IV) + intrathecal colistin therapy due to recurrent ventriculitis associated with ventriculoperitoneal (VP) shunt. But the condition of the patient worsened under this treatment and then colistin-resistant Klebsiella pneumoniae (CoR-KP) was isolated from cerebrospinal fluid (CSF) culture. Finally, she could be successfully treated with intravenous and intrathecal amikacin use.

\section{Case}

37-year-old female patient that was placed VP shunt due to congenital hydrocephalus 35 days ago; this patient admitted to our emergency with three days of headache, nausea, vomiting, blurred vision and fever. On physical examination, the patient was conscious, cooperative and her fever was $39.1^{\circ} \mathrm{C}$ and initially she did not have stiff neck. The CSF culture was obtained and meropenem $3 \times 2 \mathrm{~g}$ and vancomycin $2 \times 1$ gr IV were initiated, the signs of shunt associated ventriculitis were seen in cranial computer tomography (CT). Extraventricular drainage (EVD) was removed and shunt catheter was inserted. In the CSF samples, Pseudomonas spp, Enterobacter cloaca IBL (+), Acinetobacter baumannii and ESBL (+) Klebsiella pneumoniae were isolated respectively and many different anti-microbial agents such as meropenem, amikacin, colistin, cefoperazone/ sulbactam, piperacillin tazobactam were given at different duration (course). EVD was replaced a total of 10 times during six months, due to carbapenem resistant Acinetobacter baumannii and CR-KP [ertapenem MIC>16 Colistin MIC $=0.75 \mathrm{mcg} / \mathrm{mL}$ ] agents which isolated from CSF culture and even with intravenous and intrathecal colistin therapy which were combined with other antibiotics, we could not achieve clinical improvement in the patient during 75 days of treatment. On the $80^{\text {th }}$ days of the admission, CoR-KP (Colistin MIC $=64 \mathrm{mcg} / \mathrm{mL}$ ) were isolated from CSF cultures (Figure 1), and on the same day, IV therapy $(1 \mathrm{~g} /$ day) and intrathecal (1x30 mg) amikacin was added to the therapy, stopping colistin. On follow up; the patient was extubated from the ventilator on the $3^{\text {th }}$ days of the amikacin, CSF culture was become negative on the $7^{\text {th }}$ days of the

${ }^{\star}$ Correspondence to: Abdurrahman Kaya, Department of Infectious Diseases, İstanbul Training and Research Hospital, Istanbul, Turkey, Tel: +90 506 6113328; E-mail: dr.abdkaya@hotmail.com

Key words: Carbapenem-resistant, intrathecal, colistin

Received: October 10, 2019; Accepted: November 28, 2019; Published: December 02, 2019 
Kaya A (2019) A case of recurrent ventriculitis associated with Colistin-Resistant Klebsiella pneumoniae in patient with ventriculoperitoneal shunt treated with intrathecal amikacin

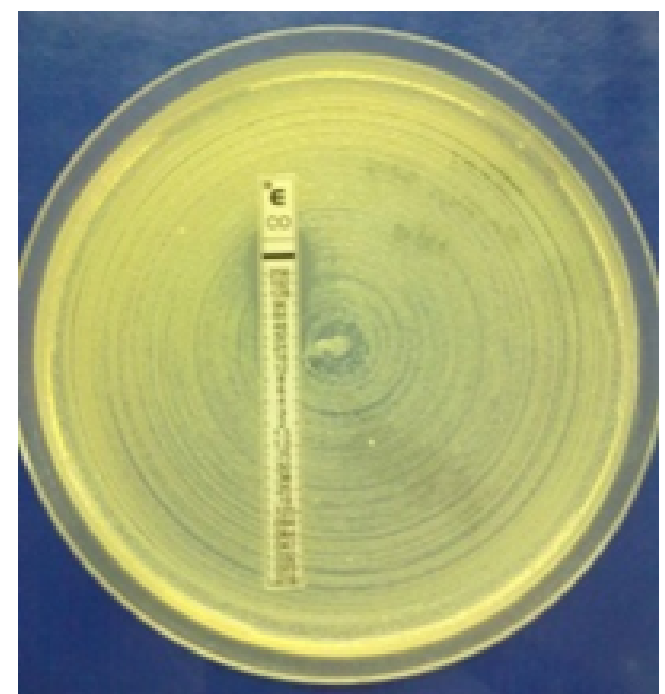

Figure 1. CoR-KP strains isolated from CSF culture on the $75^{\text {th }}$ day of the treatment $(\mathrm{MIC}=64 \mathrm{mcg} / \mathrm{mL})$

therapy and consciousness of the patient was opened on the $15^{\text {th }}$ days of the amikacin and Glasgow coma score (GCS) was increased (improved) from $9 / 15$ to $14 / 15$ and subsequently the patient was discharged from intensive care unit for regular physiotherapy and supportive treatment on the $25^{\text {th }}$ days of the cure , ultimately she returned to normal life with the recent therapies.

\section{Discussion}

Antibiotics use, especially both in medicine and in agriculture have been increasing and improperly and unnecessarily using them has deeply threatened to our life due to the emergence of resistance and this resistance makes therapy difficult to cure diseases. Because resistant bacteria are not susceptible to many potent agents and available used agents for resistant bacteria are more toxic and less potent. Recently, there has been a paucity in novel antimicrobial to treat the resistant pathogens [3]. Therefore, we will face with the worst clinical issues in the future and we should immediately take measures for this issue. These are antibiotic stewardship, control and prevention measure, hand hygiene, surveillance, cohorting of infected/colonized patients, implementation of contact precautions [4]. Particularly in nosocomial infections, these resistant microorganisms can spread from person to person, can colonize and later cause infections and outbreak $[5,6]$.

There are few options in the treatment of carbapenemase-producing organisms that is unclear and has not been reached a consensus yet [4] but if the patient has severe infection such as bacteremia and meningitidis, as in this patient. We can use two or more antibiotics due to high mortality rate and a major concern in emergence of new resistance [4] Therefore, we used combination therapy in the index case and the challenge of our case was meningitidis. Because antibiotics cannot penetrate well into the meninges, and thus the treatment become difficult to achieve improvement. Consequently, we used combination therapy with intrathecal use that is formed local antimicrobial effects. The concentration rate of the antibiotic at the inflamed site increases and this local effect, thereby facilitating the healing of the disease

It is not known how the mechanism(s) of colistin resistance is in carbapenem-resistant Enterobacteriaceae [7] and the criteria of resistance has not been well defined by the formal establishment While the preferred test for detecting colistin resistance is the broth microdilution method, E-testing also ensure us results that are compatible with the broth test [8]. Hence, we utilized the methods to determine colistin susceptibility testing and the MIC of colistin resistance was detected as $64 \mathrm{mcg} / \mathrm{mL}$.

In Around 2000 years, colistin resistance was fairly rare but along with administering in other resistant bacteria including Acinetobacter baumannii and Pseudomonas spp, resistance in colistin has begun to appear much more. while some investigator are thought that previously colistin use, and the duration of the colistin are important factors for the emergence of resistance [9-11] a report could not find any association because they found infections caused by CoR-KP but without prior colistin use [12]. In our case, the patient previously took colistin therapy for infections, we thought the bacteria might become resistant because of this use. Also, we did not have any other patient that colonized/infected with CoR-KP in this unit.

\section{Conclusion}

As a conclusion, resistant bacteria seem to increase and pose a threat to our life in the future. Thus, infection control measures should be taken immediately. In these infections, along with the use of systemic antibiotics, intrathecal use should be administered for meningitidis.

\section{References}

1. Gales AC, Reis AO, Jones RN (2001) Contemporary assessment of antimicrobial susceptibility testing methods for polymyxin B and colistin: review of available interpretative criteria and quality control guidelines. J Clin Microbiol 39: 183-190.

2. Dellit TH, Owens RC, McGowan JE, Gerding DN, Weinstein RA, et al. (2007) Infectious Diseases Society of America and the Society for Healthcare Epidemiology of America guidelines for developing an institutional program to enhance antimicrobial stewardship. Clin Infect Dis 44: 159-177.

3. Falagas ME, Kasiakou SK, Saravolatz LD (2005) Colistin: the revival of polymyxins for the management of multidrug-resistant gram-negative bacterial infections. Clin Infect Dis 40: 1333-1341

4. John Quale M, Denis Spelman M (2015) Overview of carbapenemase producing gramnegative bacilli.

5. Hossain A, Ferraro MJ, Pino RM, Dew RB, Moland ES, et al. (2004) Plasmid-mediated carbapenem-hydrolyzing enzyme KPC-2 in an Enterobacter sp. Antimicrob Agents Chemother 48: 4438-4440.

6. Leavitt A, Navon-Venezia S, Chmelnitsky I, Schwaber MJ, Carmeli Y (2007) Emergence of KPC-2 and KPC-3 in carbapenem-resistant Klebsiella pneumoniae strains in an Israeli hospital. Antimicrob Agents Chemother 51: 3026-3029.

7. MacLaren GM, Denis Spelman M (2015) Colistin: An overview.

8. Arroyo LA, Garcia-Curiel A, Pachon-Ibanez ME, Llanos AC, Ruiz M, et al. (2005) Reliability of the E-test method for detection of colistin resistance in clinical isolates of Acinetobacter baumannii. J Clin Microbiol 43: 903-905.

9. Antoniadou A, Kontopidou F, Poulakou G, Koratzanis E, Galani I, et al. (2007) Colistinresistant isolates of Klebsiella pneumoniae emerging in intensive care unit patients: first report of a multiclonal cluster. J Antimicrob Chemother 59: 786-790.

10. Lee J, Patel G, Huprikar S, Calfee DP, Jenkins SG (2009) Decreased susceptibility to polymyxin B during treatment for carbapenem-resistant Klebsiella pneumoniae infection. J Clin Microbiol 47: 1611-1612.

11. Zarkotou O, Pournaras S, Voulgari E, Chrysos G, Prekates A, et al. (2010) Risk factors and outcomes associated with acquisition of colistin-resistant KPC-producing Klebsiella pneumoniae: a matched case-control study. J Clin Microbiol 48: 2271-2274.

12. Chen S, Hu F, Zhang X, Xu X, Liu Y, et al. (2011) Independent emergence of colistinresistant Enterobacteriaceae clinical isolates without colistin treatment. J Clin Microbiol 49: 4022-4023.

Copyright: (C2019 Kaya A. This is an open-access article distributed under the terms of the Creative Commons Attribution License, which permits unrestricted use, distribution, and reproduction in any medium, provided the original author and source are credited. 